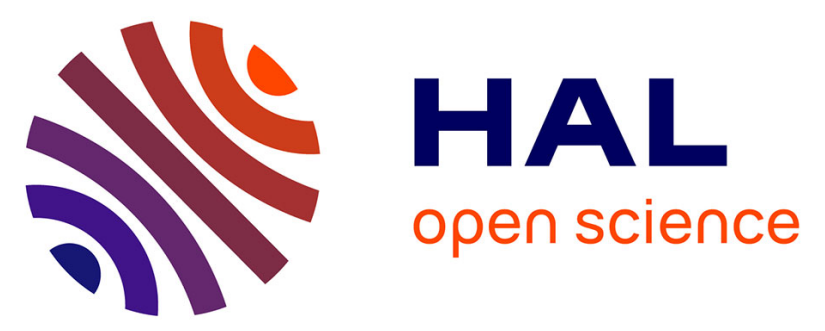

\title{
On pinning-depinning and microkink-flow in solid state dewetting: Insights by in-situ ESEM on Al thin films
} Stefan Werner Hieke, Marc-Georg Willinger, Zhu-Jun Wang, Günther H.S. Richter, Dominique Chatain, Gerhard Dehm, Christina Scheu

\section{- To cite this version:}

Stefan Werner Hieke, Marc-Georg Willinger, Zhu-Jun Wang, Günther H.S. Richter, Dominique Chatain, et al.. On pinning-depinning and microkink-flow in solid state dewetting: Insights by in-situ ESEM on Al thin films. Acta Materialia, 2019, 165, pp.153-163. 10.1016/j.actamat.2018.11.028 . hal-01941639

\section{HAL Id: hal-01941639 \\ https://hal-amu.archives-ouvertes.fr/hal-01941639}

Submitted on 29 Jan 2020

HAL is a multi-disciplinary open access archive for the deposit and dissemination of scientific research documents, whether they are published or not. The documents may come from teaching and research institutions in France or abroad, or from public or private research centers.
L'archive ouverte pluridisciplinaire HAL, est destinée au dépôt et à la diffusion de documents scientifiques de niveau recherche, publiés ou non, émanant des établissements d'enseignement et de recherche français ou étrangers, des laboratoires publics ou privés. 


\section{On pinning-depinning and microkink-flow in solid state dewetting: Insights by in-situ ESEM on Al thin films}

$\underline{\text { Stefan Werner Hieke }}^{a}$, Marc-Georg Willinger, ${ }^{b, c, d}$, Zhu-Jun Wang ${ }^{b, d}$, Gunther Richter ${ }^{\mathrm{e}}$, Dominique Chatain ${ }^{\dagger}$, Gerhard Dehm ${ }^{\mathrm{a}}$ and Christina Scheu ${ }^{\mathrm{a}, \mathrm{g},{ }^{*}}$

a Max-Planck-Institut für Eisenforschung GmbH, D-40237 Düsseldorf, Germany

${ }^{\mathrm{b}}$ Fritz Haber Institute of the Max-Planck-Society, 14195 Berlin, Germany

${ }^{c}$ Max-Planck-Institute of Colloids and Interfaces, Department of Colloid Chemistry, Research Campus Golm, 14424 Potsdam, Germany

${ }^{d}$ now at: Scientific Center for Optical and Electron Microscopy, ETH Zürich, 8093 Zürich,

Switzerland

${ }^{\text {e }}$ Max-Planck-Institut für Intelligente Systeme, 70569 Stuttgart, Germany

${ }^{\dagger}$ Aix-Marseille Univ, CNRS, CINaM, Marseille, France

g Materials Analytics, RWTH Aachen University, Kopernikusstr. 10, 52074 Aachen, Germany

${ }^{*}$ Corresponding author; Max-Planck Institut für Eisenforschung GmbH

Max-Planck Str. 1

40237 Düsseldorf

Germany

Phone: +49 2116792720

Fax: +492116792360

Email: s.hieke@mpie.de, willinger@fhi-berlin.mpg.de, marc.willinger@scopem.ethz.ch, zhujun@fhi-berlin.mpg.de, richter@is.mpg.de, chatain@cinam.univ-mrs.fr, g.dehm@mpie.de, c.scheu@mpie.de 


\section{Abstract}

The dynamics of solid state dewetting phenomena of a $50 \mathrm{~nm}$ thick, mazed bicrystalline Al film on single crystalline $\alpha-\mathrm{Al}_{2} \mathrm{O}_{3}$ (sapphire) substrates was studied in-situ using an environmental scanning electron microscope (ESEM). The bicrystalline Al thin films served as a model system where the influence of grain boundaries and texture effects are well determined compared to polycrystalline films. The experiments were performed in controlled oxidizing and reducing atmospheres at $773 \mathrm{~K}$ and $823 \mathrm{~K}$, respectively, to shed light on the differences in dewetting mechanisms and dynamics. While the reducing atmosphere led to spontaneous dewetting at $823 \mathrm{~K}$ after an incubation time of a few minutes, a hierarchical dewetting process was observed for the sluggish dewetting under oxidizing conditions. Voids initiated at (substrate or surface) defects and expanded trying to maintain a hexagonal shape. Pinning and depinning processes led to a discontinuous void growth and irregular void shapes including finger instabilities. As a consequence, the void growth followed a variety of power law exponents between 0.10 and 0.55 . A new microkink-flow mechanism was discovered at the terminating Al planes at the void.

Keywords: In situ; Solid state dewetting; Thin films; Capillarity phenomena; Interface diffusion 


\section{Introduction}

Interpretation of dynamics on post-mortem samples is challenging due to the loss of time resolution which may obscure the actual interplay of the dominating mechanisms $[1,2]$. In-situ techniques allow the direct observation of time-dependent phenomena, e.g. layer growth, grain growth, oxidation or phase transformation and reveal the details of the involved mechanisms [1, 3-5]. In the present work in-situ environmental scanning electron microscopy (ESEM) was used to investigate the dynamics of the solid state dewetting behavior of mazed bicrystalline $\mathrm{Al}$ thin films on sapphire under well-defined atmosphere and temperature conditions.

Solid state dewetting is usually controlled by surface diffusion and capping layers can be added on thin films in order to suppress dewetting [6, 7]. However, material transport via short-circuit pathways below the passivating layer can occur [8] and lead to nanowire growth [9]. Void formation despite the presence of capping layers is known as a degradation phenomenon for $\mathrm{Al}$ conductor lines on $\mathrm{SiO}_{2}$ passivated $\mathrm{Si}$ [10]. Thermally induced stresses were identified as driving forces for dewetting with grain boundary diffusion as the major diffusion path [11, 12]. In a recent work, we have studied post-mortem the formation of annealing-induced voids within $<111>$ textured (several grain orientations) $250 \mathrm{~nm}$ thick Al thin films on single crystalline sapphire [13]. Voids formed via retraction of the Al thin film underneath the continuous oxide layer. Grain boundary and interface diffusion were the dominating diffusion pathways while surface diffusion was suppressed [8, 13]. Irregular void shapes indicated a discontinuous void growth mechanism [8]. Temperature induced void formation of $\mathrm{Al}$ thin films covered with a capping layer was observed in literature for $\mathrm{Al}$ on passivated Si [12, 14] and Al on single crystalline sapphire [8, 13, 15, 16]. All mentioned studies analyzed the solid state annealing behavior indirectly post-mortem without direct observation of the involved processes, except for a study of Sugano et al. [17]. They observed the annealing induced void formation in Al thin films by optical microscopy in-situ on the $\mu \mathrm{m}$ level [17].

Solid state dewetting of thin films without capping layers was observed by various insitu techniques including electrical resistance measurements [18], Auger electron spectroscopy [19], low-energy electron microscopy [20], laser light transmission [21, 22], confocal laser microscopy [23, 24], atomic force microscopy [25], X-ray diffraction [13, 26, 27], time resolved differential reflectometry [28], scanning electron microscopy (SEM) [1, 29] and transmission electron microscopy (TEM) [2, 30, 31]. High spatial resolution is only achieved by SEM or TEM, but for these methods the observed processes are usually limited to (ultra)high vacuum conditions.

This work reports direct observations of the dynamics of the solid state dewetting behavior of bicrystalline, nominal $50 \mathrm{~nm}$ thick Al films on sapphire in oxidizing atmosphere 
using an ESEM. The Al thin film possesses a heteroepitaxial orientation relationship with the single crystalline substrate with the close packed planes and directions parallel to each other, which will be termed OR a/b in this article: OR a/b $\{111\} \pm<\overline{1} 10>_{\mathrm{Al}} \|(0001)<10 \overline{1} 0>_{\mathrm{Al}_{2} \mathrm{O}_{3}}$ ( \pm indicates the symmetrically equivalent twin variants $\mathrm{a}$ and $\mathrm{b}$ obtained by a $180^{\circ}$ rotation around the $<111>$ surface normal). The mazed bicrystalline Al thin films serves as a defined model system to reduce the interference of texture evolution and different grain boundary types as observed for oligocrystalline or polycrystalline thin films [13, 26]. The mechanisms of annealing induced void formation, growth and faceting as well as finger instabilities and their evolution with time were tracked in-situ and further analyzed. A microkink-flow mechanism is observed for the first time in-situ. Dewetting under reducing atmosphere was also investigated to emphasize the impact of the surface oxide on the dynamics.

\section{Experimental}

\subsection{Thin film growth}

Thin films were grown in a molecular beam epitaxy system (DCA instruments, Finland) with a base pressure of $4 \cdot 10^{-7} \mathrm{~Pa}$. (0001) oriented $\alpha-\mathrm{Al}_{2} \mathrm{O}_{3}$ two inch wafers (miscut $<0.25^{\circ}$, one side polished, thickness $330 \pm 25 \mu \mathrm{m}$, CrysTec Kristalltechnologie, Berlin, Germany) were used as substrates. $\mathrm{Ar}^{+}$-sputtering (200 eV, $5 \mathrm{~min}$ ) was applied within the molecular beam epitaxy system to remove contaminations and structural defects were healed by subsequent annealing at $1273 \mathrm{~K}$ for $60 \mathrm{~min}$. The nominal film thickness and deposition rate were $50 \mathrm{~nm}$ and $0.03 \mathrm{~nm} / \mathrm{s}$, respectively. During deposition, the sapphire substrate was rotated and kept at room temperature.

\subsection{In-situ annealing and microstructural characterization}

In-situ isothermal annealing experiments were performed in a field emission gun (FEG) ESEM FEI Quanta 200 equipped with a heating stage and a gas supply unit (mass flow controllers from Bronkhorst) [32]. Four short time $(<1 \mathrm{~h})$ and a single long-term $(\sim 15.3 \mathrm{~h})$ annealing experiments have been carried out (full datasets can be found in Supplementary movie 1 and Supplementary movie 2). The solid state dewetting experiments were performed in oxidizing atmosphere $\left(\mathrm{O}_{2}\right.$, total pressure $\left.\sim 30 \mathrm{~Pa}\right)$ at $773 \mathrm{~K}$ and at $823 \mathrm{~K}$ in reducing atmosphere $\left(67 \% \mathrm{H}_{2} / 33 \% \mathrm{~N}_{2}\right.$, total pressure $\left.\sim 50 \mathrm{~Pa}\right)$ atmosphere. All gases possessed a purity of $99.99 \%$. The base pressure within the ESEM chamber prior annealing was below $5 \cdot 10^{-3} \mathrm{~Pa}$. Temperatures were measured with a K-type thermocouple, that was mechanically clamped onto the sample. Secondary electron micrographs were acquired at an acceleration voltage of $5 \mathrm{kV}$ using an Everhart-Thornley detector. Heating of the sample to the target temperatures of $773 \mathrm{~K}$ and $823 \mathrm{~K}$ as well as the selection of the region of interest for isothermal annealing experiments took up to $15 \mathrm{~min}$. The areas displayed in this article are 
representative as controlled by additional micrographs recorded after annealing at different locations. Comparing irradiated to non-irradiated regions, no impact of the electron beam was found. The in-situ setup allowed fast cooling (estimated cooling time down to $473 \mathrm{~K}$ $\sim 30$ seconds) and hence quenching of the microstructure.

Prior to the in-situ experiment and post-mortem, the samples were characterized by SEM and electron backscatter diffraction (EBSD) using FEG SEMs (Jeol JSM 6500F and Zeiss Merlin) or focused-ion-beam (FIB) systems (FEI Helios Nanolab 600 or 600i dual beam SEM/FIB). The microstructure of the as-deposited film was also characterized using $X$-ray diffraction (XRD) in a GE Seifert diffractometer equipped with a $\mathrm{Cu}$ X-ray source $\left(K_{\alpha}=1.54 \cdot 10^{-10} \mathrm{~m}\right)$. The grain size was estimated by the line intercept method with horizontal straight lines using the TSL OIM software applied on post-mortem EBSD data. This is an apparent grain size (mean value \pm standard deviation) due to the mazed microstructure of interpenetrating twinned grains. For the as-deposited film the grain size was determined from cross-sectional TEM samples.

Statistical void size data was obtained from post-mortem plan-view SEM micrographs and is presented by a mean value \pm standard deviation of the equivalent void radius (only voids with an area $>10 \mu \mathrm{m}^{2}$ considered). Individual voids were tracked during the in-situ experiment. The perimeter and the equivalent void radius as a function of time were extracted from the in-situ SEM datasets by image processing using ImageJ [33] including the plugin suite FeatureJ [34] and the plugin Template Matching and Slice Alignment [35].

The velocities of facets and microkinks were determined by measuring the position with respect to a reference position (temporal resolution $17.7 \mathrm{~s}$ for the short-term dataset shown in

Figure 2 , $88.5 \mathrm{~s}$ for the long-term dataset shown in Figure 6).

The error of the displacement $x$ is estimated with two times the pixel size. For facets, the normal was constructed for the analyzed facets for a reference frame and the position along the normal was tracked. Diffusion constants $D$ for Al were derived by analyzing the mean square displacement $x^{2}$ of facets/microkinks as a function of time $t\left(x^{2}=2 D t\right)$. The measured values are apparent diffusion constants as discussed in section 0.

Site-specific cross-sectional specimens for TEM analysis were prepared by FIB using a modified procedure of the lift out technique reported in literature [36-38]. More details and the parameters of the applied method, briefly described here, can be found in [8]. A Pt layer was deposited to protect the specimen surface from $\mathrm{Ga}$ ion milling. After the transfer of $1-$ $2 \mu \mathrm{m}$ thick TEM lamellae to a TEM grid, the sample was thinned to electron transparency with accelerating voltages from $30 \mathrm{kV}$ down to $5 \mathrm{kV}$ to minimize beam damage. The final ion beam current at $5 \mathrm{kV}$ was set to $15 \mathrm{pA}$. 
TEM characterization of all films was carried out using a FEI Titan Themis $60-300 \mathrm{X}$ FEG S/TEM instrument operated at $300 \mathrm{kV}$, equipped with a probe corrector for spherical aberration. For scanning TEM (STEM) measurements a spot size of $0.15 \mathrm{~nm}$ and a convergence semi-angle of the electron probe of $23.8 \mathrm{mrad}$ were used. Probe currents of $\sim 70 \mathrm{pA}$ were applied for imaging and up to $400 \mathrm{pA}$ for energy-dispersive $\mathrm{X}$-ray spectroscopy (EDS) measurements. EDS experiments, were carried out in STEM mode using a FEI Super$X$ windowless EDS system with 4 synchronized silicon drift detectors attached to the Titan Themis. A high angle annular dark field (HAADF) detector covering the angular range of collection semi-angles from 73 to 352 mrad was used for imaging. High signal to noise ratios for the atomically resolved STEM HAADF micrographs, were obtained by stacking of up to 60 frames using the Smart Align software [39]

\section{Results}

\subsection{In-situ microstructural evolution}

\subsubsection{Discontinuous void growth mechanism}

The initial, dense, homogenous Al film possessed defects like scratches and surface contamination in the form of aggregates. They were visible at a few locations and were used as markers during the in-situ experiments.
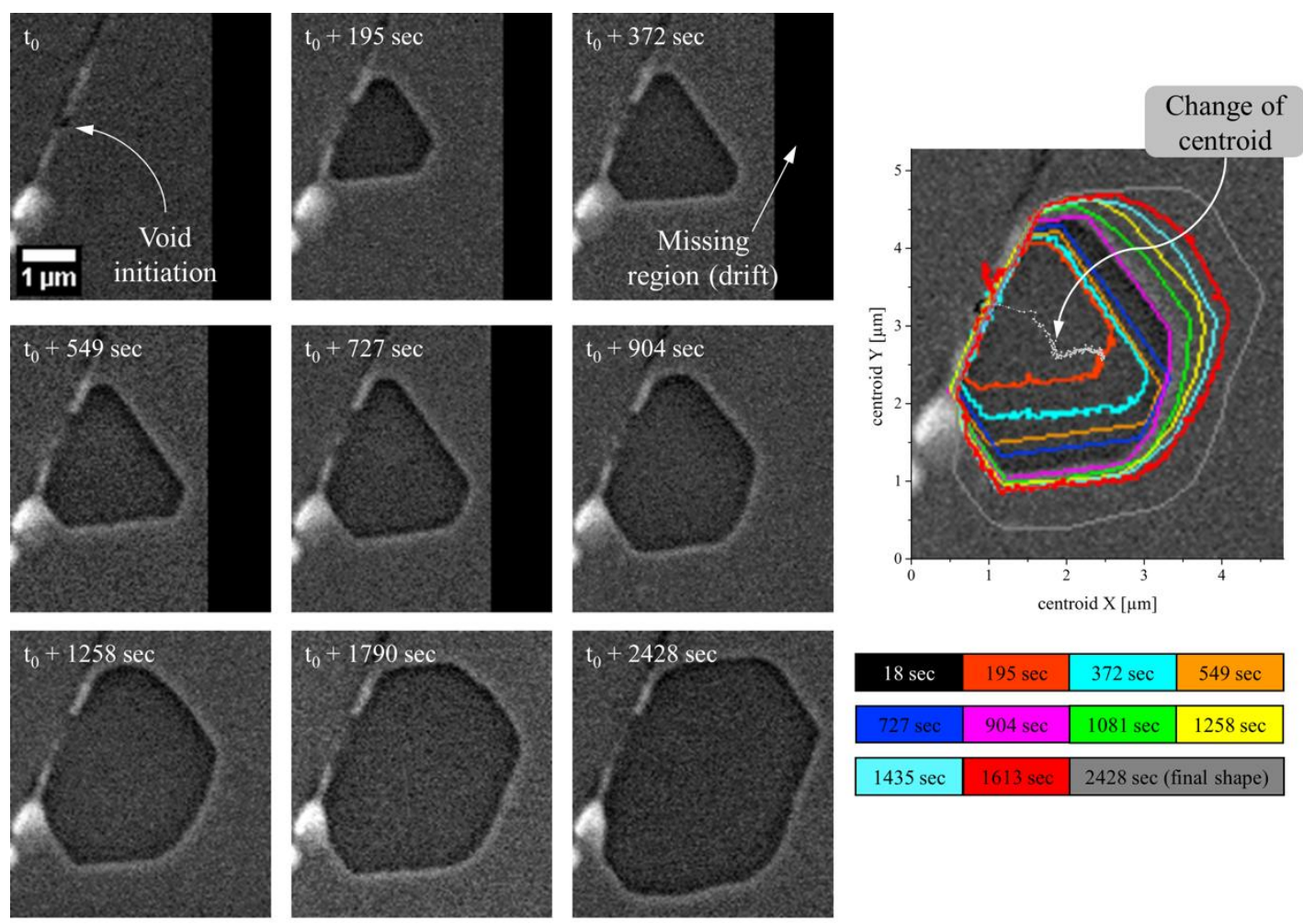

Figure 1 Left: Void evolution with annealing time observed in-situ in the ESEM ( 40 min, $\mathrm{O}_{2}$, $\sim 773 \mathrm{~K}$. A part of the micrographs is missing due to sample drift. The full region of interest can be seen in Supplementary figure 1 and the complete dataset is shown in the 
Supplementary movie 1). Right: Superposition of the perimeter after selected annealing times and variations of the void centroid with annealing time.

The in-situ SEM micrograph sequence in

Figure 1 shows the discontinuous evolution of a void with annealing time ( $40 \mathrm{~min})$. The void initiates close to a defect (scratch) in the Al film and opens with a hexagonal shape with alternating short and long facets. As the void grows its shape deviates from the hexagon due to pinning of the triple line at various locations, e.g. defects. Pinning can eventually be overcome, and Al film retraction continues. As a consequence of pinning the growth speed varies from place to place and the voids adopt asymmetric shapes. A typical evolution of the shape is shown in

Figure 1 (right) as overlay of the void perimeter for different annealing times (background SEM micrograph for $t_{0}+904 \mathrm{~s}$ ). The change of boundary conditions which results in $a$ variation of the growth direction can be visualized by a change of the centroid of the void with annealing time (

Figure 1 right). The centroid of this void moves by $\sim 1.7 \mu \mathrm{m}$ which is large compared to its maximum dimension of $\sim 5.0 \mu \mathrm{m}$. The void grows towards a direction away from the pinning obstacles, which is in this case the scratch on the top left side.
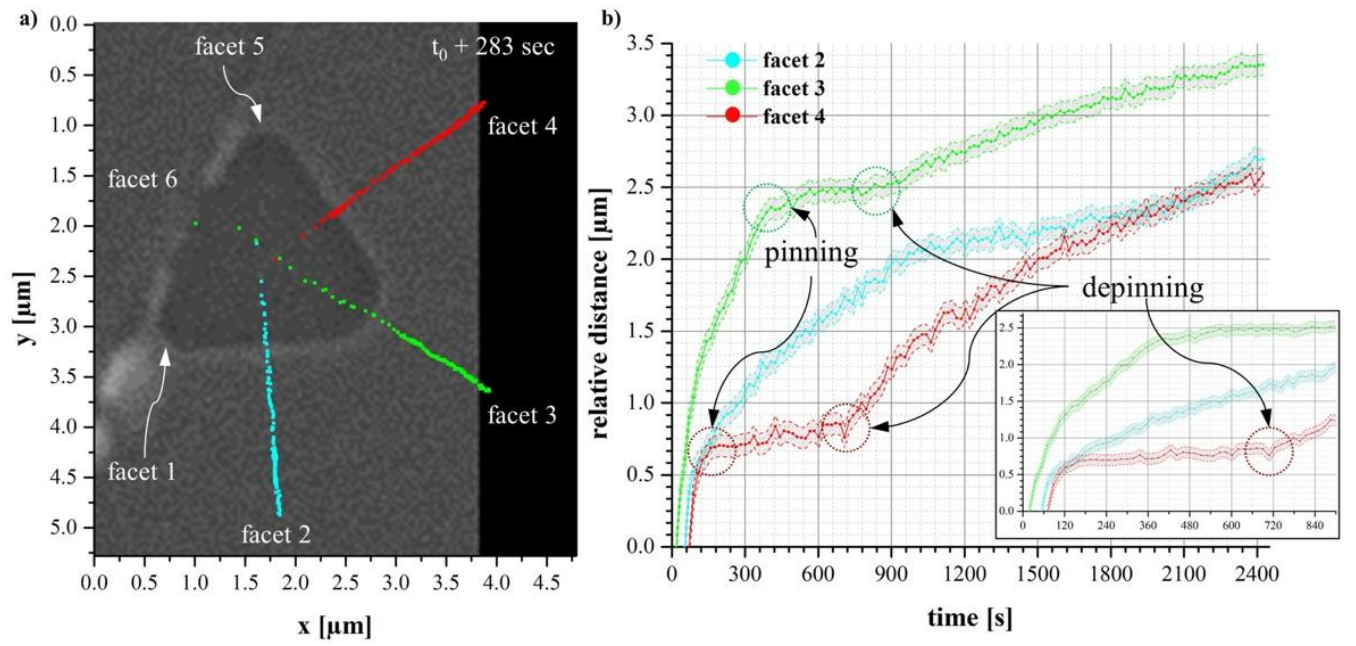

Figure 2 a) Overlay of the positions along the normal of the facet as a function of time on the reference frame at $t_{0}+283 \mathrm{~s}$. b) The relative distance as a function of time for the facets 2,3 and 4 reveals a discontinuous Al film retraction. Inset shows a zoom-in between 0 and $900 \mathrm{~s}$.

These side facets of the void which do not interfere with the scratch were tracked (see facets 2, 3 and 4 in

Figure 2a) and their position changes are shown as colored lines in

Figure 2 $a$. The positions along the facet normal of a reference frame $\left(t_{0}+283 \mathrm{~s}\right)$ are plotted in

Figure $2 b$. The irregular void growth and shift of the centroid prevented the tracking of all facets from the first frames and the identification of a unique starting point. Thus, the relative distance of each track is shown, whereas the first detected point serves as the relative 
starting point. Pinning events and the subsequent depinning at later stages can clearly be observed for facets 3 and 4 (

Figure $2 b)$. At the beginning of the void growth, a faster diffusion was observed. For facet 3 , the shortest facet, a maximum value of $\sim 8 \cdot 10^{-15} \frac{\mathrm{m}^{2}}{\mathrm{~s}}$ was measured for the first frames. For the longer facets 2 and 4 , the initial points were not tracked, but estimated from a straight line from the point where the void initiated to the facet position at $t_{0}+283 \mathrm{~s}$. The diffusion constant for them was in the range $\sim 4 \cdot 10^{-15} \frac{\mathrm{m}^{2}}{\mathrm{~s}}$. An average diffusion constant of $\sim 1-2 \cdot 10^{-}$ $15 \frac{\mathrm{m}^{2}}{\mathrm{~s}}$ was found for all moving facets.

\subsubsection{Void growth kinetics}

The velocity of the retracting facets decreases as the hole grows. For example, in the case of void 1 (

Figure 1 ), the average velocity of the moving facets $2-4$ is in the range of $\sim 0.5-1 \frac{\mathrm{nm}}{\mathrm{s}}$ for the main growth regime. In the initial stages, facet velocities up to $\sim 15 \frac{\mathrm{nm}}{\mathrm{s}}$ were observed depending on the pinning sites (e.g. Void 1 in Supplementary figure 1a).
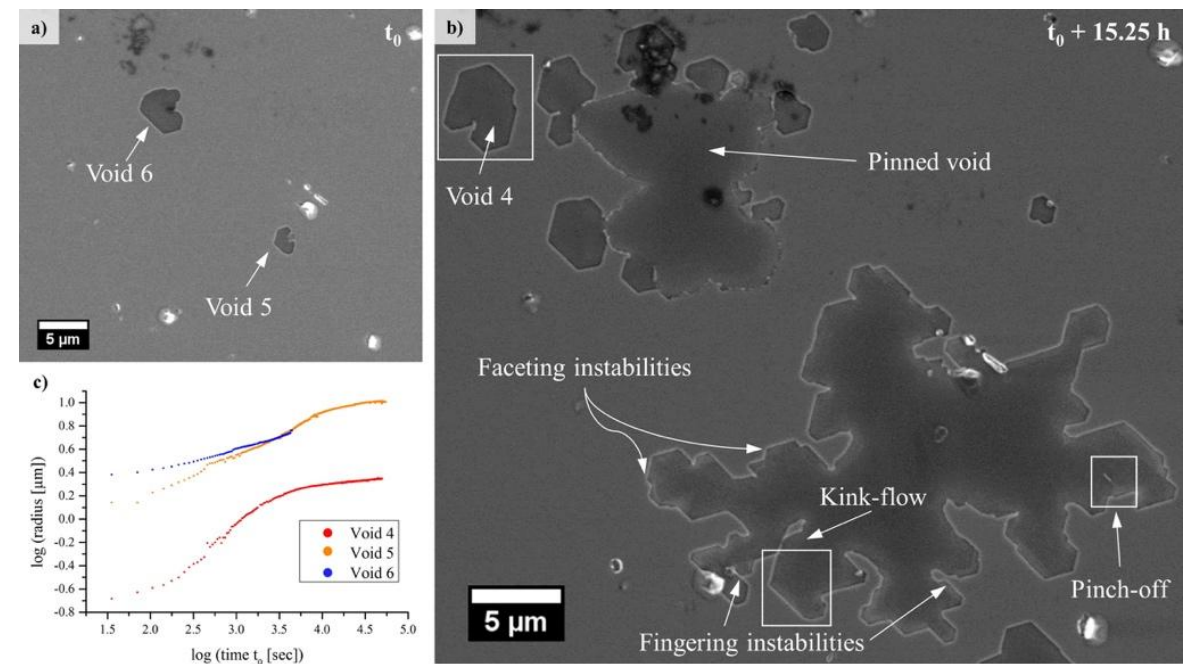

Figure 3 a) Microstructure of the region of interest for the long-term in-situ annealing experiments after reaching the final temperature $\sim 773 \mathrm{~K}$. b) Temperature induced microstructural changes after $\sim 15.3 \mathrm{~h}$ annealing $\left(\mathrm{O}_{2}, \sim 773 \mathrm{~K}\right.$, see text for details, the full dataset can be seen in the Supplementary movie 2). The evolution of different features with time is indicated by the white rectangles. c) Equivalent void radius evolution with annealing time for the voids 4 to 6 (Note: for void 4 the time of the initial void formation was set as $t_{0}$, for void 5 and 6 , the absolute time was used because the initial void formation was not observed).

The equivalent void radius $r_{\text {void }}$ can be described by a power law function of the annealing time $t: r_{\text {void }} \sim t^{x}$. The power law fit applies for the main growth regime. Different power law exponents were extracted for different voids tracked in the same dataset: $r_{\text {void } 1} \sim$ $\mathrm{t}^{0.36}, \mathrm{r}_{\text {void } 2} \sim \mathrm{t}^{0.26}$ and $\mathrm{r}_{\text {void } 3} \sim \mathrm{t}^{0.11}$ and they stay constant for short-term experiments 
(Supplementary figure 1). Some voids are already present when reaching the target temperature of $773 \mathrm{~K}$ indicating that they form during heating up (e.g. voids 5 and 6 in
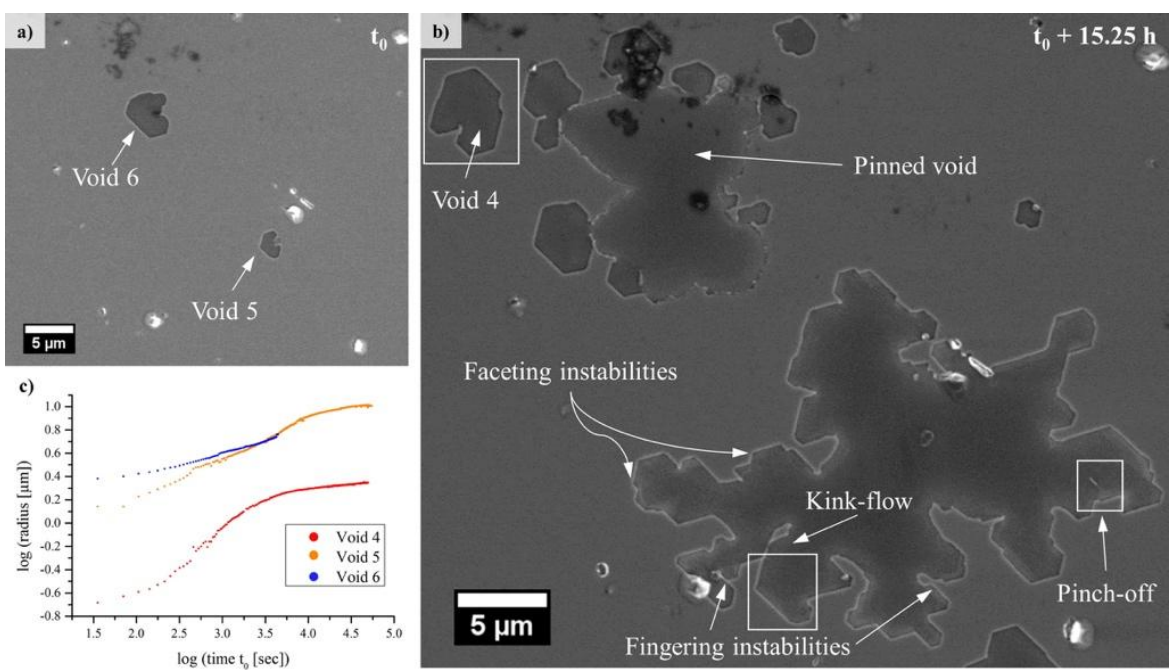

Figure 3). In addition, new voids form during the isothermal treatment after various incubation times (e.g. void 4). In the long-term experiment, the growth of individual voids slows down (void 4 and 5) or even stops. This can be seen in the change of the slope in the log-log-plot

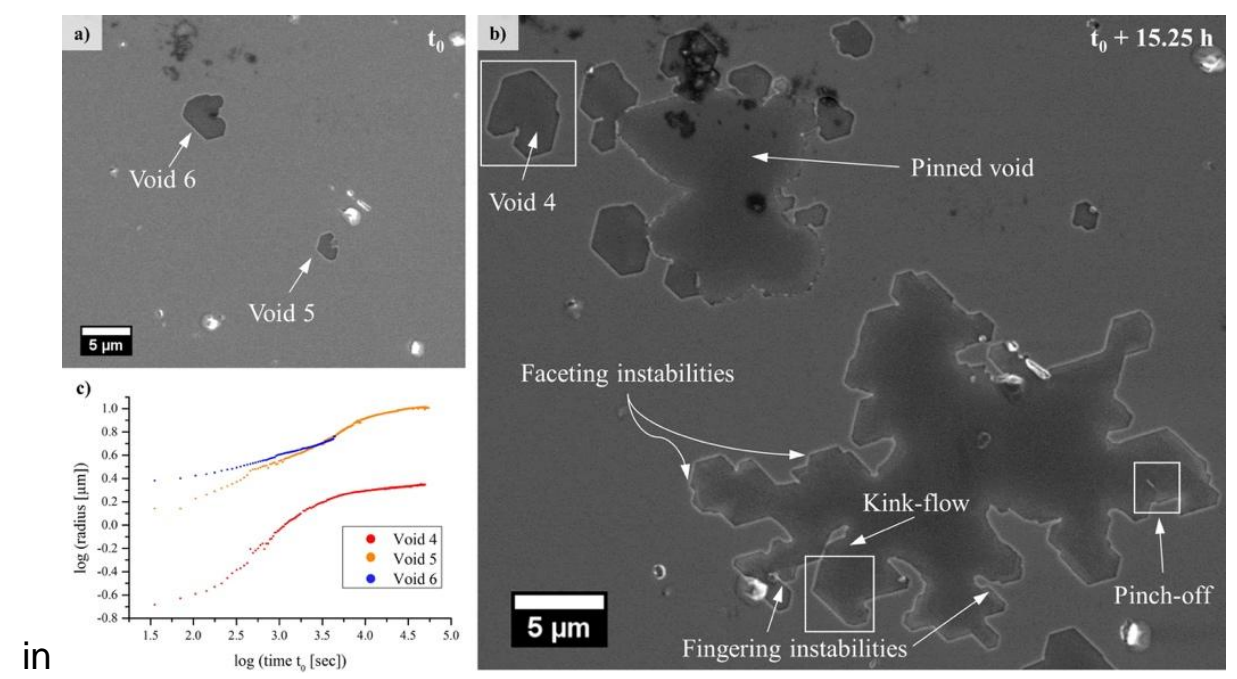

Figure 3c. In summary, the power law exponent $x$ of the individual voids varies from 0.10 and 0.55 depending on the pinning of the retracting film at obstacles.

\subsubsection{Formation of microkinks and fingers}

Varying boundary conditions during void growth causes the formation of complex void morphologies. Faceting instabilities or microfaceting, finger instabilities, and pinch-off processes occur as a result of pinning as shown in Figures $3-7$ and Supplementary movie 2 for the long-term experiment ( 15.3 h). Pinning and depinning of a retracting Al front during 
the dewetting process is shown for void 4 in the SEM image sequence (only selected frames) and the perimeter evolution of the void is displayed in Figure 4 (see also Supplementary movie 2). Coincidentally to fingers, faceting occurs in the right part of the void shown in Figure 4.
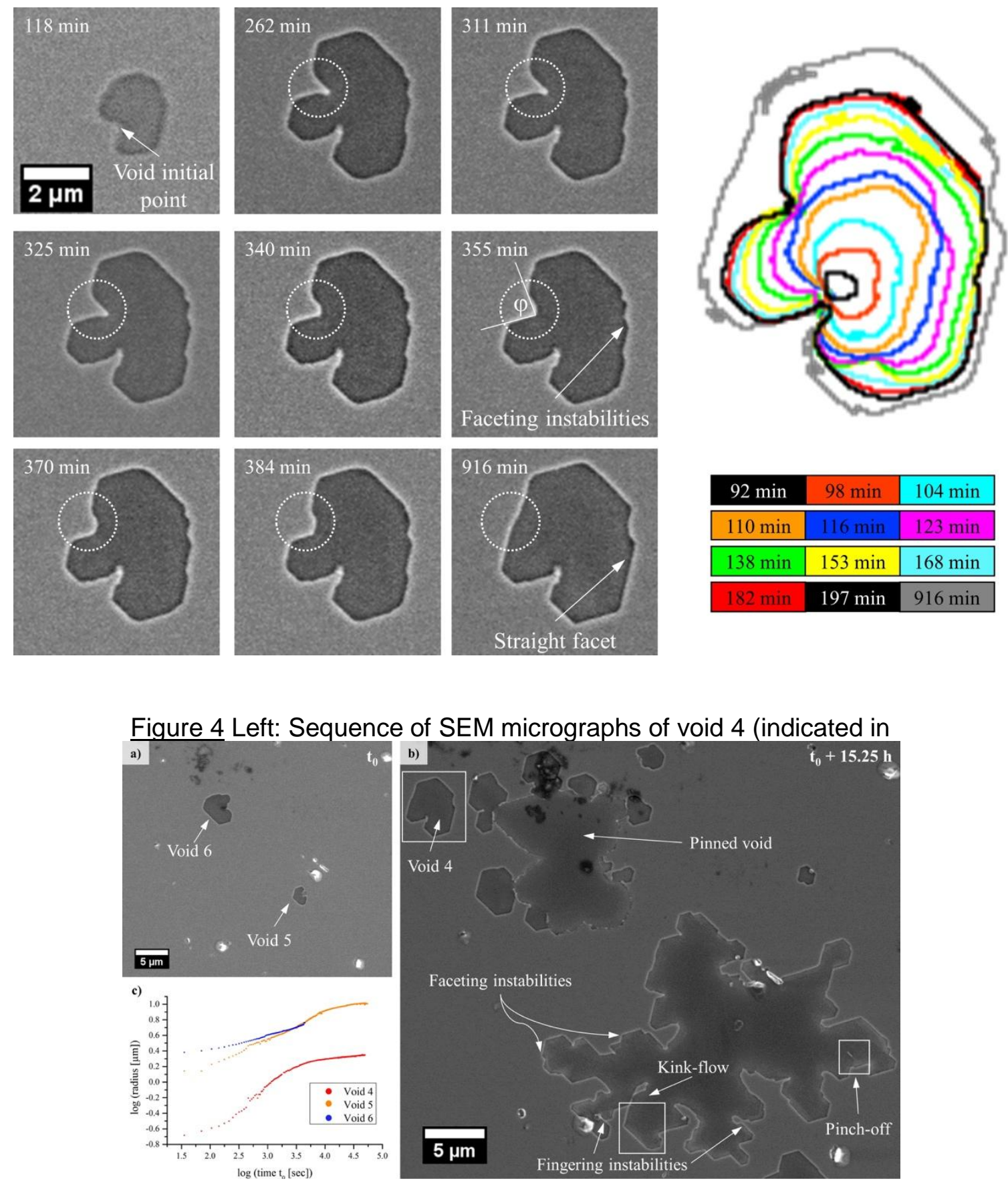

Figure 3) revealing pinning and subsequent depinning for distinct regions of the retracting $\mathrm{Al}$ film. Faceting instabilities are observed for different annealing times. Right: The superposition of the void perimeter is shown for selected annealing times for the main growth phase.

Figure 5 shows an unexpected microkink-flow diffusion mechanism along void facets resulting in film retraction and (micro)facets. Supplementary figure 2 shows the size of the 
microkinks into the void which is in the range from $\sim 20 \mathrm{~nm}$ to $300 \mathrm{~nm}$ (max. $\sim 800 \mathrm{~nm}$ ) in post-mortem SEM plan-view micrographs. The position of the microkink along the facet was tracked as a function of time and is shown as overlay on the SEM micrograph in Figure $6 a$ where the first and last frame, where the microkink was observed, were merged. Within the experiment the microkink moved back and forth, once each in this sequence, removing two "steps/rows" of the facet. The relative distance to the intersection of the facet with the adjacent one at $t_{0}+351$ min (reference position) as a function of time $t$ is shown in Figure $6 b$. For both motions, forward and backward, the first steps could not be resolved because of limits in spatial and/or time resolution. During the turning back, a jump of the microkink occurred ( 620 nm in $885 \mathrm{~s}$ ). The motion of the microkink is discontinuous on a local scale due to pinning. The average backward motion occurs faster $\left(\bar{v}_{\text {back }} \sim 0.16 \frac{\mathrm{nm}}{\mathrm{s}}\right)$ compared to the forward motion $\left(\bar{v}_{\text {for }} \sim 0.06 \frac{\mathrm{nm}}{\mathrm{s}}\right)$. During the jump, the extrapolated velocity $\bar{v}_{\text {jump }} \sim 0.70 \frac{\mathrm{nm}}{\mathrm{s}}$ is a magnitude higher. The analysis of the backward motion (jump excluded) revealed an average Al|void interface diffusion constant of $\sim 2 \cdot 10^{-16} \frac{\mathrm{m}^{2}}{\mathrm{~s}}$, whereas the apparent diffusion constant varied from $\sim 4 \cdot 10^{-17}$ to $3 \cdot 10^{-16} \frac{\mathrm{m}^{2}}{\mathrm{~s}}$ on a local time scale. For the forward motion an average apparent diffusion constant of $\sim 8 \cdot 10^{-17} \frac{\mathrm{m}^{2}}{\mathrm{~s}}$ with local variations from $\sim 2 \cdot 10^{-17}$ to $3 \cdot 10^{-}$ $16 \frac{\mathrm{m}^{2}}{\mathrm{~s}}$ was calculated.
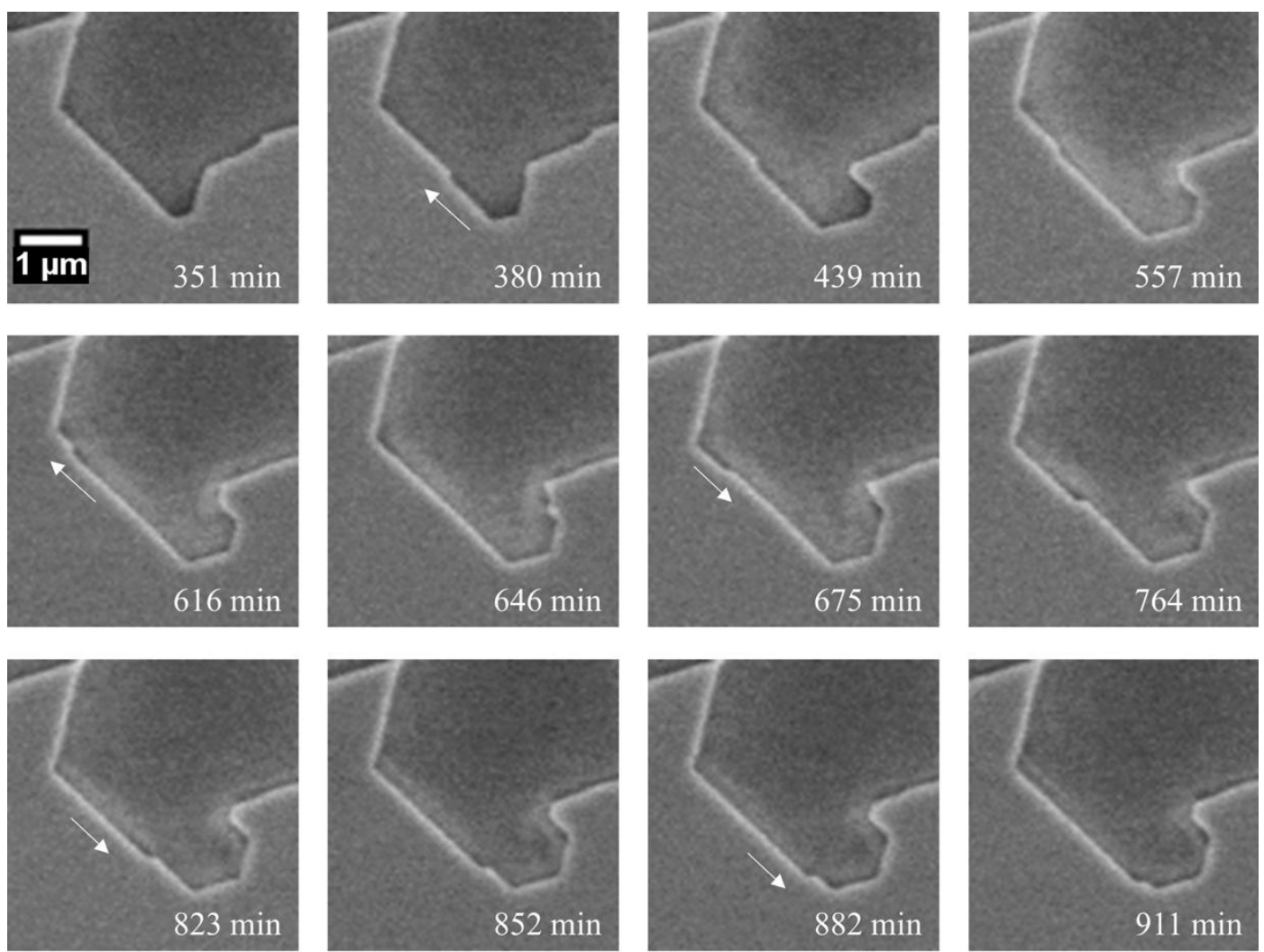
Figure 5 The solid state dewetting mechanism of a large void via a microkink-flow process along the Al sidewall facet is shown in the sequence of SEM micrographs of the marked

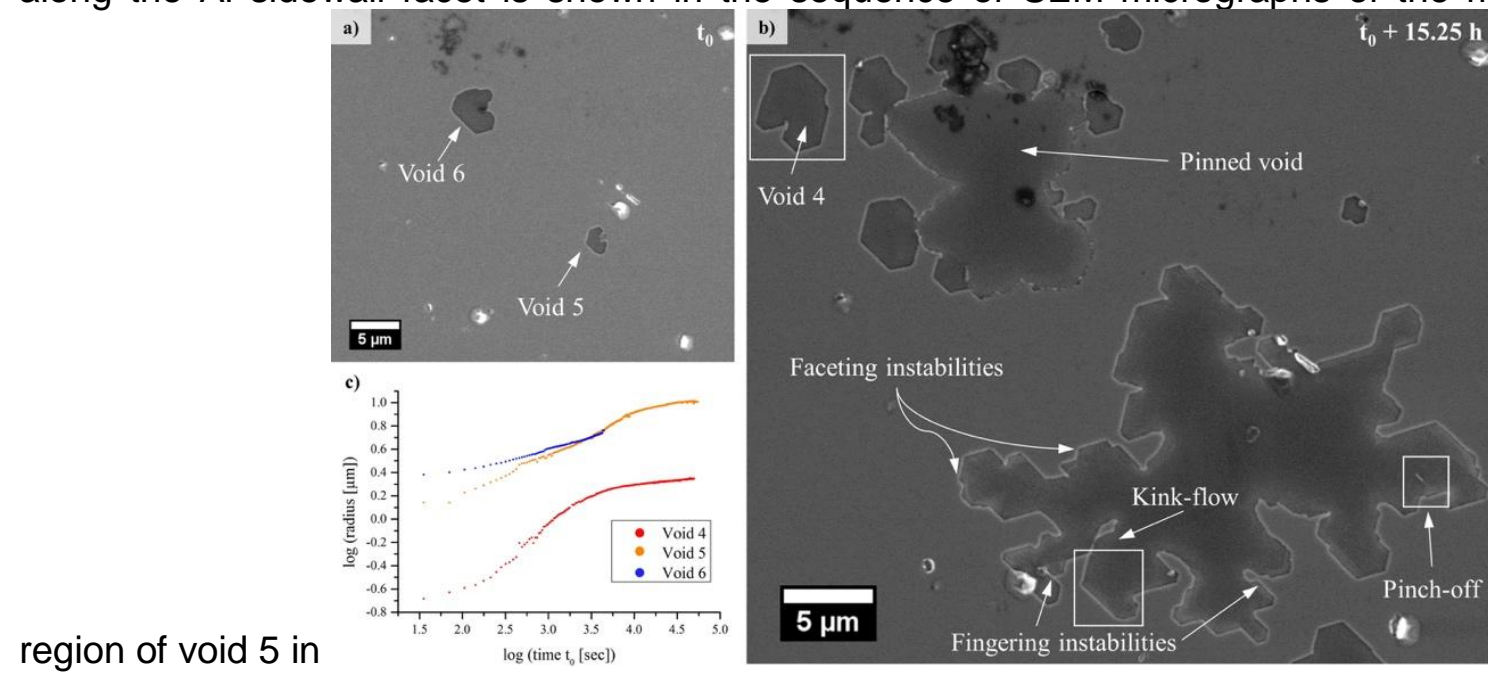

\section{Figure 3.}

a)

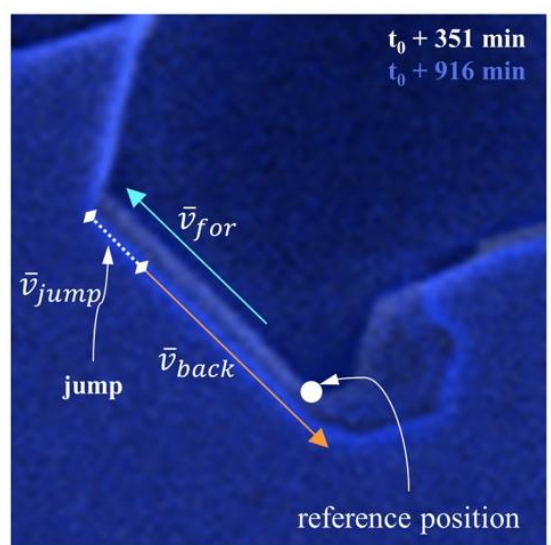

b)

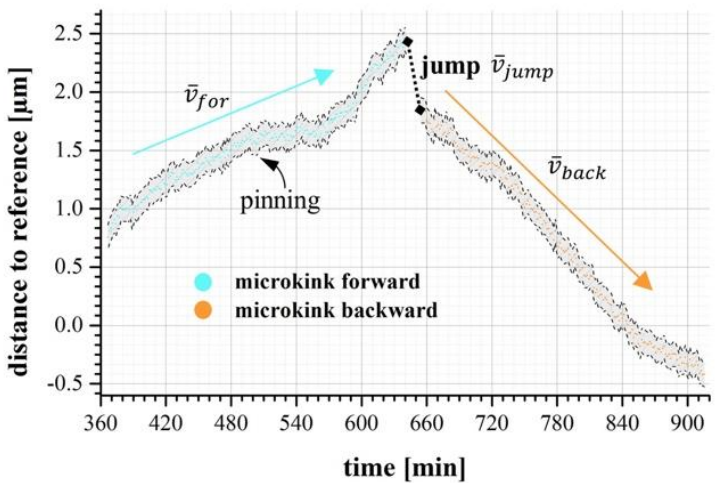

Figure 6 a) Overlay of the positions of a microkink moving back and forth along the facet as a function of time on the merged, color-coded SEM frames at $t_{0}+351 \mathrm{~min}$ (Al|void interface in white) and $t_{0}+916$ min (Allvoid interface in intense blue). b) The relative distance as a function of time for both microkink motion reveal a discontinuous motion on a local scale affected by pinning and different local velocities. Note the different magnitude of time compared to

Figure $\underline{2}$. 

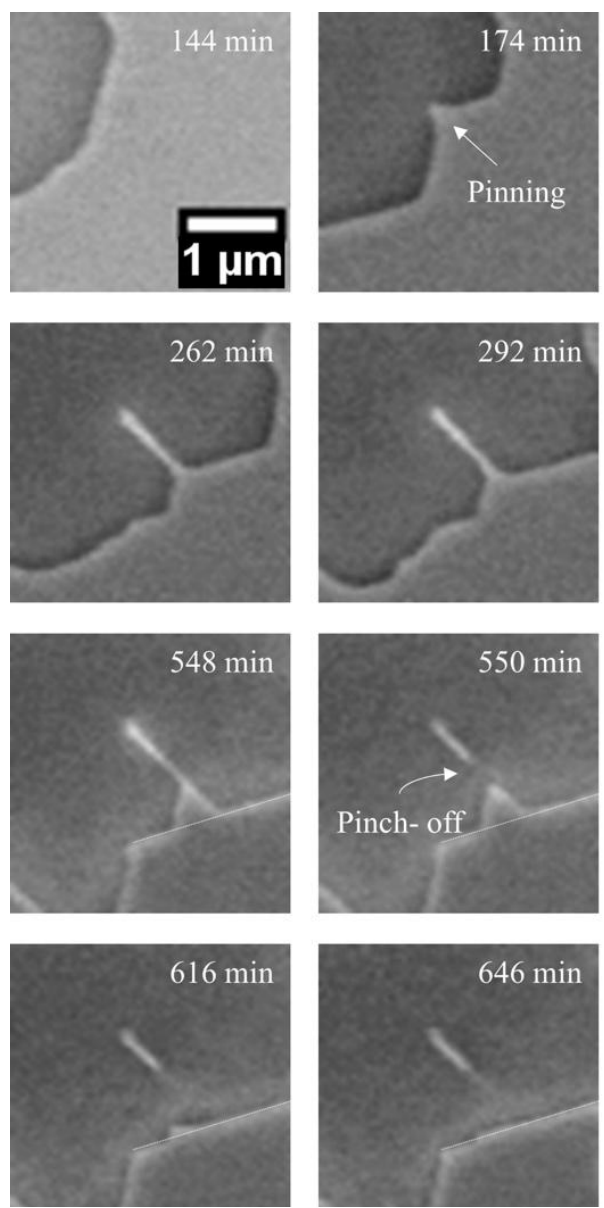
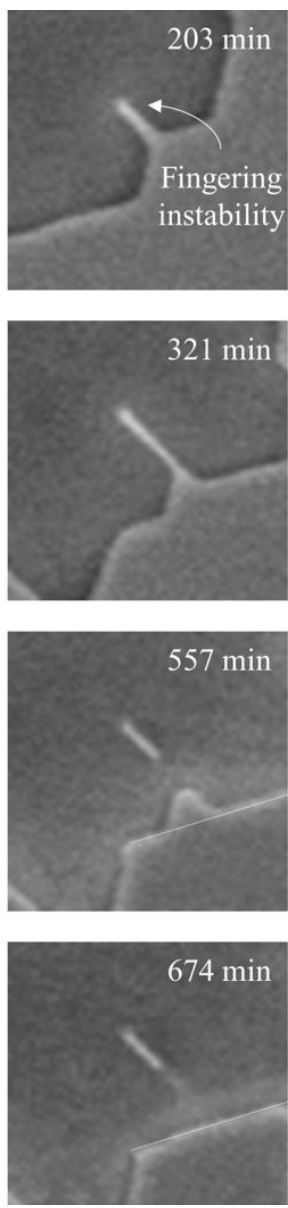
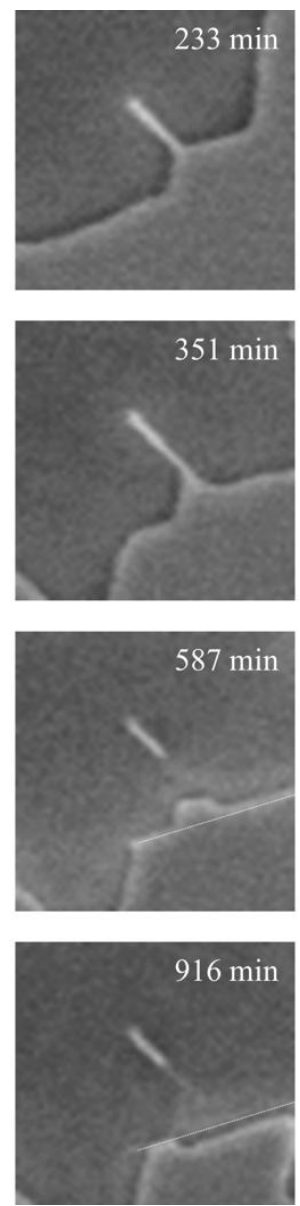

Figure 7 The formation of an isolated finger is revealed in the sequence of SEM micrographs
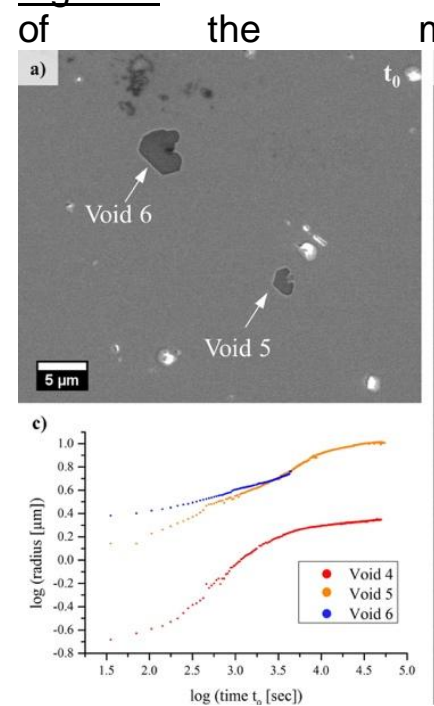
marked region
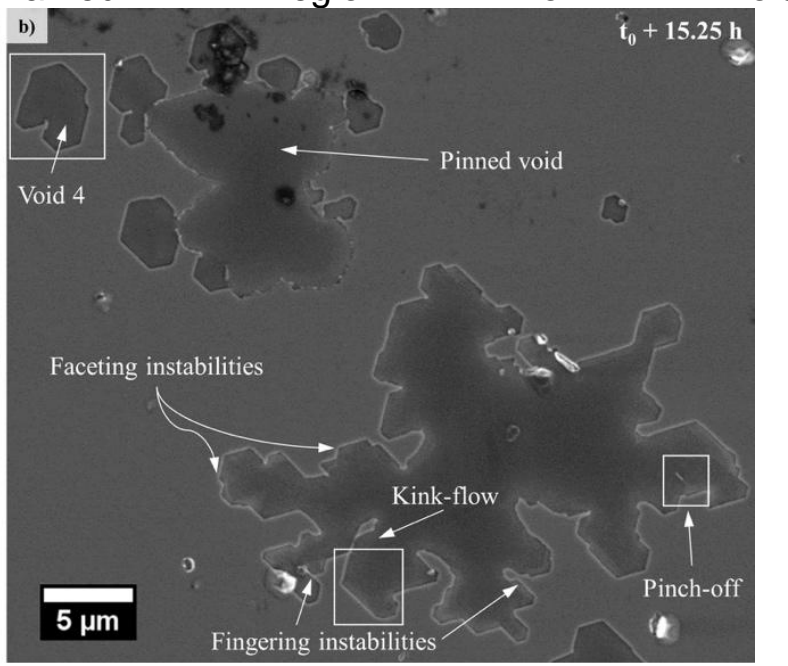

Figure 3. Pinning of the retracting Al thin film and subsequent retraction at both sides of the pinned region leads to the formation of a finger. With increasing annealing time a pinch-off of the finger is observed and the adjacent, now disconnected Al thin film forms a straight facet.

Both phenomena, formation of a finger and faceting, are observed in the SEM micrograph sequence in Figure 7. As the Al film adjacent to a pinning point retracts, a finger builds up and lasts for $\sim 300 \mathrm{~min}$. The adjacent Al film possesses distinct facets with increasing time while faceting instabilities occur due to the microkink-flow mechanism 
described above. Within a recorded image frame (acquisition time $\sim 2 \mathrm{~min}$ ), the finger pinches off and forms an isolated thin line. The base of the former finger is unstable and is subsequently removed. The removal of the base and the formation of a defined facet occurs

relatively fast ( $<100 \mathrm{~min}$ ) compared to the depinning of the finger in Figure 4 ( $500 \mathrm{~min})$. The resulting Al film becomes straighter. The dewetting process continues via the microkink-flow mechanism along the newly formed facet resulting in faceting instabilities. Alternatively, depinning of complete fingers can also occur as observed in Figure 4. The curvature of the marked Al film finger increases. The curvature is described by the angle $\varphi$ between the two feature facets which decreases from $\sim 120^{\circ}$ down to $\sim 41^{\circ}$ when the pinning condition is overcome and the Al film retraction continues. A second pinning event occurred at the lower Allvoid interface in Figure 4, but is overcome despite a comparably high angle $\varphi$ of $\sim 109^{\circ}$ (Supplementary movie 2).

\subsubsection{In-situ annealing in reducing atmosphere}

In-situ annealing at $\sim 823 \mathrm{~K}$ in reducing atmosphere $\left(67 \% \mathrm{H}_{2} / 33 \% \mathrm{~N}_{2}, \sim 50 \mathrm{~Pa}\right)$ led to a different solid state dewetting behaviour compared to the one observed when annealing in an oxidizing atmosphere. After dwell times in the range of minutes once the desired temperature has been reached, the film breaks-up into isolated, $\mu$ m-sized features within the Al film and regions with exposed substrate. The process is relatively fast: at $823 \mathrm{~K}$ it takes less than $35 \mathrm{~s}$. Supplementary figure 3 shows the resulting, complex microstructure which is beyond the scope of this article. However, these experiments in reducing conditions confirm that the sluggish void formation, diffusion-limited dewetting and pinning induced discontinuous void growth, as observed in an oxidizing atmosphere, is due to the presence of a stable and continuous surface oxide discussed for the ex-situ annealing experiment [8].

\subsection{Comparison of the microstructure before and after annealing}

\subsubsection{Initial microstructure after deposition}

Figure $\underline{8} \mathrm{a}$ and $\mathrm{d}$ show the twin structure with vertical grain boundaries and an average width of the grains in the range from $50-200 \mathrm{~nm}$ for the as-deposited thin film. The $\{111\}$ Al pole figure in

Figure $\underline{8} b$, obtained by $\mathrm{XRD}$, revealed $\mathrm{a} \sim 1: 1$ ratio between the two twinned in-plane orientations $\mathrm{OR} a / b$ in accordance to literature [40]. The Al thin film is covered by an amorphous and continuous native, $3.6 \pm 0.4 \mathrm{~nm}$ thick $\mathrm{Al}_{2} \mathrm{O}_{3}$ oxide layer (

Figure $\underline{8} \mathrm{c}$ ). The high interface contrast is due to $\mathrm{Ga}$ enrichment at the $\mathrm{Al} \mid \mathrm{Al}_{2} \mathrm{O}_{3}$ interfaces which happened during the FIB TEM sample preparation as reported in literature [13, 41, 42]. 


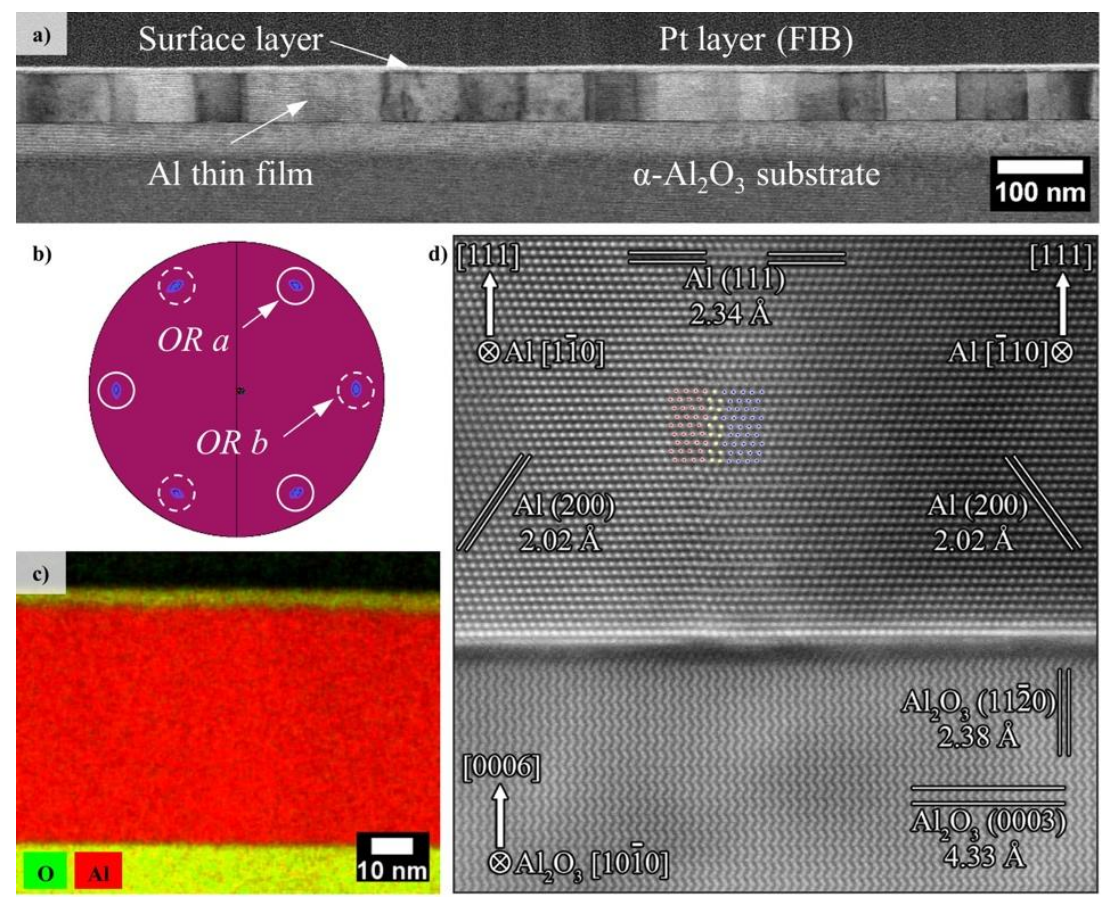

Figure 8 The initial microstructure of the as-deposited Al thin film. a) Cross-sectional STEM $\mathrm{BF}$ micrograph revealing the columnar grain structure of the Al thin film. b) $\{111\}$ XRD pole figure with the two twin-related growth variants OR a/b marked. c) Combined Al- and O-EDS map showing a uniformly thick Al film covered by a continuous surface oxide layer. g) Atomic resolved STEM HAADF micrograph of a triple phase boundary at the Al|substrate interface

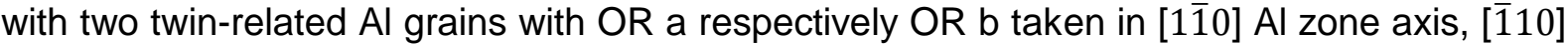
Al zone axis and [10 $\overline{1} 0] \mathrm{Al}_{2} \mathrm{O}_{3}$ zone axis.

\subsubsection{Microstructure after in-situ annealing}

Several in-situ annealed samples $\left(\mathrm{O}_{2}, \sim 773 \mathrm{~K}\right)$ were investigated post-mortem. Postmortem EBSD analysis of short-term as well as of long-term in-situ annealed samples reveal no change in texture (Supplementary figure 4). After annealing, the Al twin growth variants OR $a / b$ are still arranged in a mazed structure, as reported for some fcc metallic thin films on single crystalline (0001) $\alpha-\mathrm{Al}_{2} \mathrm{O}_{3}$ for certain growth conditions [40, 43-46]. Upon annealing the grain size increases to $1.7 \pm 1.4 \mu \mathrm{m}$ (short-term, $\sim 40 \mathrm{~min}$ ) and 1.9 $\pm 1.5 \mu \mathrm{m}$ (long-term, $\sim 15.3 \mathrm{~h}$ ) which is a factor of $\sim 10$ compared to the as-deposited thin films. The stagnation of grain growth is in accordance to literature observations [13]. Plan-view SEM micrographs after $\sim 15.3 \mathrm{~h}$ in-situ annealing at $773 \mathrm{~K}$ reveal that the voids occupy $\sim 3.9 \%$ of a $\sim 381 \times 286 \mu \mathrm{m}$ large area (Supplementary figure 5).

For TEM analysis, selected regions of the short-term ( 40 min) in-situ annealed sample containing voids as well as finger instabilities were prepared in cross-section by FIB (Figure 9a, Supplementary figure 6). Both, the Al film and the void, are capped by a surface oxide layer $5.5 \pm 0.4 \mathrm{~nm}$ thick. The voids underneath a continuous surface oxide membrane appear dark in the SEM micrographs. After a collapse of the surface membrane, the oxide layer is in contact with the substrate as marked in Figure 9a and voids appear bright in plan- 
view SEM micrograph due to charging effects of the sapphire substrate. This was only rarely observed in-situ, but for the majority of voids post-mortem after the in-situ experiment. The collapse probably occurred after the in-situ experiment due to the small film thickness and the pressure difference between the microscope environment and atmosphere. Figure $9 \mathrm{~b}$ shows the Al|void interface region without rim at the surface triple junction. The total thickness (metal plus oxide films) remains almost constant indicating that Al redistributes over large areas [8]. The STEM micrograph in Figure $9 \mathrm{c}$ reveals that the sectioned Al finger is tapered towards the periphery. The corresponding overlaid Al-O-EDS map, shown in Figure $9 \mathrm{~d}$, confirm that the finger consists of pure Al. Neither interfacial pores nor voids are observed in the HRSTEM micrographs of regions with intact Al film. An atomically sharp Al|substrate interface is observed as for the as-deposited thin film TEM sample (Figure 9e). The high contrast at the interface is a FIB artefact as described above.
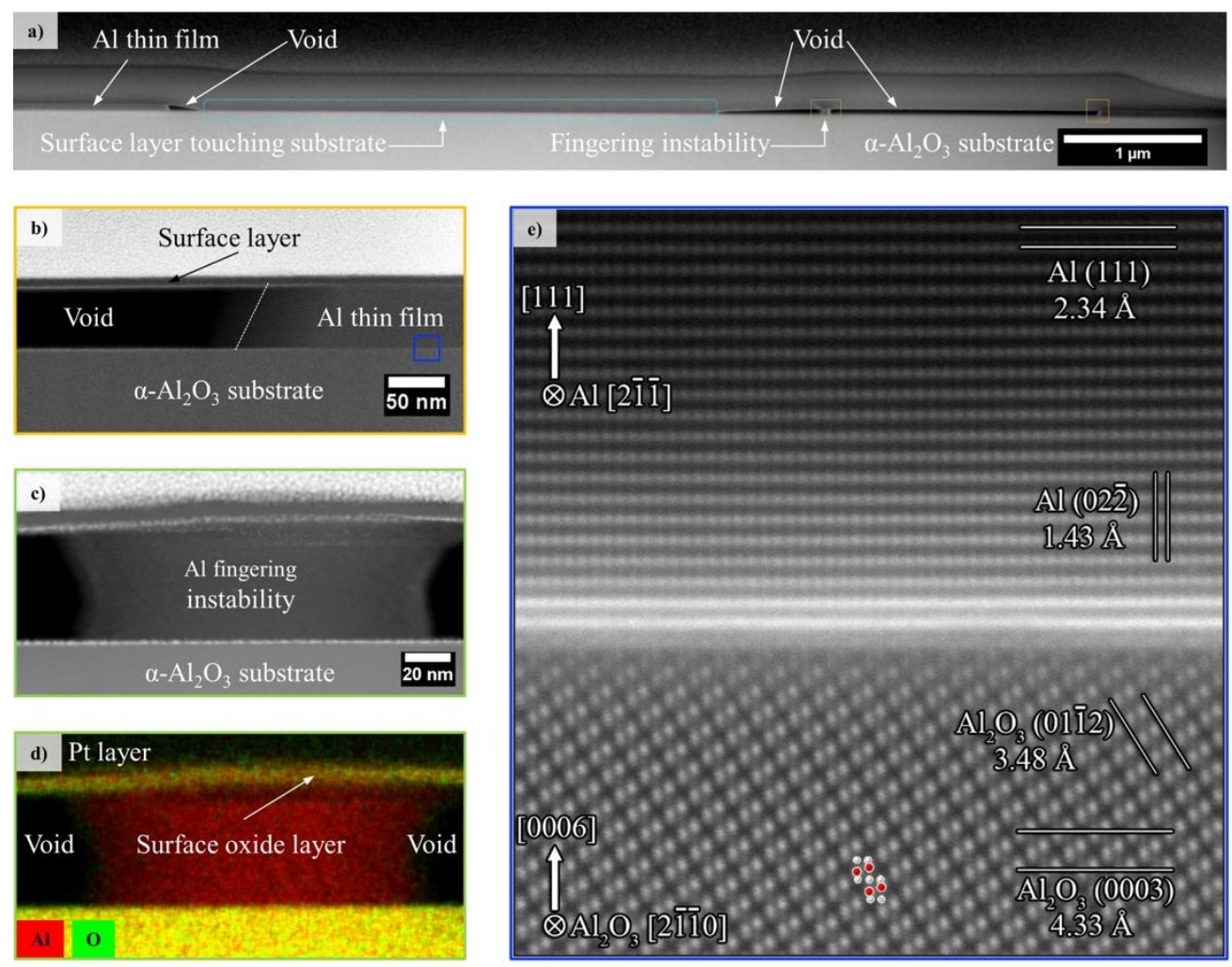

Figure 9 Annealing induced microstructural changes revealed by post-mortem analysis ( $\sim 40 \mathrm{~min}, \mathrm{O}_{2}, \sim 773 \mathrm{~K}$, corresponding in-situ movie: Supplementary movie 1). a) Crosssectional SEM micrograph of the region marked in Supplementary figure 6. Voids, a finger and a region with a collapsed surface membrane are observed. Cross-sectional STEM HAADF micrographs showing b) the Allvoid interface region with a surface membrane, continuously covering both, the Al thin film as well as the void and c) the marked finger. d) Combined EDS map of the finger region. The finger consists of pure Al covered with an intact Al oxide membrane. d) Atomic resolved STEM micrograph of a region adjacent to the Allvoid interface confirming OR $a / b$. The twin variants cannot be distinguished in the present $\mathrm{Al}$ [2 $\overline{1} \overline{1}]$ zone axis which is parallel to the $[2 \overline{1} \overline{1} 0] \mathrm{Al}_{2} \mathrm{O}_{3}$ zone axis $(\mathrm{Al}$ and $\mathrm{O}$ are marked red and white, respectively). 


\section{Discussion}

\subsection{Comparison of the solid state dewetting behavior in oxidizing and reducing conditions}

This in-situ study of $50 \mathrm{~nm}$ passivated mazed bicrystalline Al films allowed the direct observation of the dewetting behavior, as the initiation and growth of voids in oxidizing conditions. During the in-situ annealing experiments in reducing atmosphere, we speculate that cracks form in the covering oxide membrane because the Al film expands upon heating relative to the more rigid sapphire substrate. The lack of oxygen prevents healing of the cracks $[47,48]$ and outward Al diffusion becomes possible. Surface diffusion is then the dominant diffusion mechanism for Al redistribution resulting in breaking-up of the film in $\mu \mathrm{m}$ sized features on the sapphire substrate (Supplementary figure 3). In contrast, for oxidizing conditions the surface oxide is stabilized, e.g. in $\mathrm{O}_{2}$ atmosphere in the ESEM in this in-situ study or by $\mathrm{O}_{2}$ or $\mathrm{H}_{2} \mathrm{O}$ as residual gases during ex-situ annealing studies $[8,13,16]$. This is also indicated by an increase in thickness of the surface oxide while still continuously covering the film and the voids in accordance to our previous ex-situ annealing study $[8,13]$. The diffusion processes involved in void growth have been discussed in our earlier study in depth [8]. In brief, Al diffusion from the Al|void|sapphire triple junction towards the Al|void|surface oxide and subsequent outward Al diffusion at the Al|surface oxide interface was assumed as major Al diffusion pathways. Still, interfacial diffusion at the Al|sapphire interface cannot be excluded.

New voids formed after different incubation times constantly as also reported in literature [13,49-51]. In the ESEM, void initiation at surface scratches was observed in-situ. However, voids were also observed to a minor degree within regions without visible surface defects (compare Supplementary movie 2). Void formation can also start at defects at substrate imperfections at the interface to the films, which could be e.g. contaminants or surface steps being present prior Al film deposition. 2D defects like $\Sigma 3\{2 \overline{1} \overline{1}\}$ twin boundaries might also act as a starting point for void initiation. Müller and Spolenak reported that pores initially form at the film|substrate interface, grow preferentially along grain boundaries in $\mathrm{Au}$ thin films and evolve to voids [51]. In addition to the void initiation, all mentioned defects are involved in pinning events determining the evolution of the void with time as described in the next section.

\subsection{In-situ observed dynamics, pinning and microkink-flow mechanism}

Interface and grain boundary diffusion are important diffusion pathways in the present sapphire substrate|Al film|surface oxide system as discussed in [8]. Al self-diffusion constants at $773 \mathrm{~K}$ are reported in the range $\mathrm{D} \sim 4 \cdot 10^{-14} \frac{\mathrm{m}^{2}}{\mathrm{~s}}[52-54]$ which is higher than the measured ones $\left(\sim 1-2 \cdot 10^{-15} \frac{\mathrm{m}^{2}}{\mathrm{~s}}\right.$ for void 1 in 
Figure 1). The determined ones are only apparent diffusion constants as no (local) increase in film thickness was measurable. To calculate the average characteristic length $L$ a time $t$ of $40 \mathrm{~min}$ and the $D_{\text {volume }}=4 \cdot 10^{-14} \frac{\mathrm{m}^{2}}{\mathrm{~s}}$ from literature [52-54] were inserted in $L=\sqrt{2 D t}$ and a value of $L \sim 13 \mu \mathrm{m}$ was found for the short-time experiment (

Figure 1, Supplementary movie 1). This value is higher than the maximum change of position for the tracked Al|void interface of $\sim 3 \mu \mathrm{m}$. Interface diffusion is supposed to be the dominating diffusion process [8] resulting in even higher characteristic lengths $L$ compared to volume diffusion. This indicates that $\mathrm{Al}$ could be redistributed to the surrounding remaining $\mathrm{Al}$ film. A simple calculation shows that an increase of $1 \mathrm{~nm}$ in film thickness for the Al film surrounding the void is sufficient to distribute all Al contained in the volume of void 1 (Supplementary figure 1b, additional radius: $\sim 11 \mu \mathrm{m})$. However, the obtained, apparent diffusion constant can be considered as lower-bound estimate and differ for the void growth just by one order of magnitude. For a more detailed model, the influence of the newly formed surfaces and interfaces, capillary forces and especially pinning events should be considered as addressed by Amram et al. [55]. Driving forces and diffusion paths may locally vary as visible in the pinning events.

The in-situ observed void size evolution is represented by the change of the equivalent radius with annealing time $t$ (see Supplementary figure $1 \mathrm{c}$ ). It can be described by one power law $r_{\text {void }} \sim t^{x}$ for the short-term experiments ( 40 min) where $r_{\text {void }}$ is the equivalent void radius and the individual exponent $x$ for each void varies in the range from 0.10 to 0.55 . The results of the long-time $(\sim 15.3 \mathrm{~h})$ annealing experiment can only be explained by using two power law functions with two different exponents for one void which might be a result of a decreasing driving force and/or varying diffusion path.

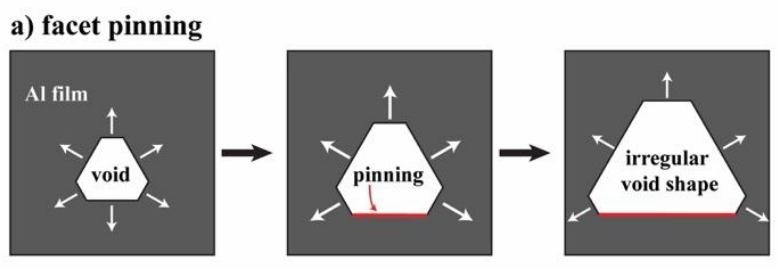

b) finger formation

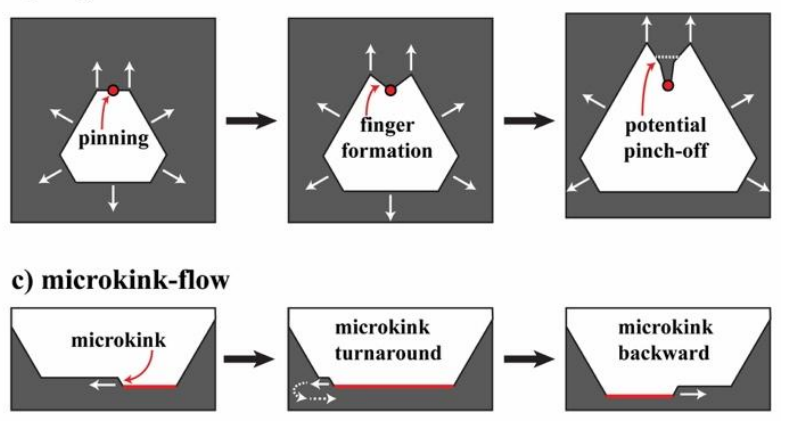

Figure 10 Sketch of pinning affected void growth mechanisms in plan-view: a) facet pinning, b) finger formation and c) microkink-flow (Al thin film in grey, void in white, pinned triple line in red, growth directions in white, further growth steps in dashed white). 
The void size evolution for ex-situ annealed $250 \mathrm{~nm}$ Al thin films containing two twinned ORs (rotated by $30^{\circ}$ relative to each other) was described by a power law $r_{\text {void }} \sim t^{0.23}$ [13]. The different exponent of the ex-situ study can be caused by statistics (many voids, but poor time resolution for the ex-situ annealing study and vice versa for an in-situ study) as well as by other parameters such as film thickness, texture, grain size (evolution), and distribution of obstacles. Especially the film thickness is known to strongly influence the solid state dewetting kinetics [6].

This in-situ study reveals discontinuous void growth mechanisms due to pinning: void facet pinning, finger formation and microkink motion (see sketch in

Figure 10). Pinning of the triple line can explain the irregular shaped voids observed in exsitu studies which deviate from the initial hexagonal void shape (

Figure 10a) [8, 16]. Depinning can additionally occur as shown in Figure 4. The degree of complexity to describe the dewetting phenomena is increasing with annealing time due to varying boundary conditions affecting the void growth and also leading to additional microstructural features, e.g. fingers and microfacets.

Pinning induces the formation of Al fingers as schematically shown in

Figure 10b. While the front around the finger recedes, the finger gets longer and may even pinch-off as observed in-situ (Figure 7). Fingers are observed in most cases parallel to the $<110>$ direction and confirm anisotropic film retraction [56]. The formation of fingers and pinch-off during solid state dewetting is found for various systems in literature $[6,21,57,58]$ and is commonly described to be caused by an unstable, retracting edge. A finger is a feature of very high curvature which instability increases as its length increases. The increasing tensile stress might cause the transverse contraction leading to a concave shape in contrast to the convex Al sidewall at the Al|void interface (Figure 9b, c). Freestanding fingers were reported as the origin of adjacent faceted particles after dewetting without capping layer [26]. Instead of pinch-off, depinning may occur as shown in Figure 4. Thus film retraction continues, the finger regresses and a facet evolves. Fingers can also completely disappear by Ostwald ripening as observed in-situ by Niekiel et al. [31]. Supplementary figure 2 shows faceting as well as finger coincidentally within the same void. In the previous ex-situ study [13] annealing of a $250 \mathrm{~nm}$ thick Al film, faceting and film retraction adjacent to a pinned position were also observed, while almost no finger formation occurred.

With increasing annealing time faceting instabilities were observed. Faceting instabilities are described in literature as a result of anisotropic edge retraction [56] which we observed directly in-situ in this study. The facet retraction occurs in average with 
$D_{\text {apparent }}=\sim 1 \cdot 10^{-15} \frac{\mathrm{m}^{2}}{\mathrm{~s}}$, until the Al film is pinned and microkink motion along the facet sets in as sketched in

Figure 10c. Subsequently, Al transport occurs along the facets in $<110>$ directions by a microkink-flow mechanism via interface diffusion at the Al sidewall, i.e. the Al|void interface. After reaching an intersecting obstacle, a straight facet has been formed again, as shown in the SEM sequences in Figure 4 and

Figure $\underline{5}$, until the microkink flow mechanism turns back. The initial stages of the microkinkflow mechanism seem to occur faster than the SEM frame time in the in-situ experiment. The obtained, apparent diffusion constants are one or two magnitudes lower compared to the facet movement. The reason for the different diffusion constants is not clear, but even at this time scale pinning events were observed. Different microkink heights ( 20 - 800 nm) were found, whereas for higher microkinks faceting could be resolved by SEM (Supplementary figure 2). The combination of a pinned microkink with one or several following microkinks pinned at the same obstacles results in an increase in microkink height and finally faceting instabilities (observed in-situ for the long-term dataset in Supplementary movie 2). Postmortem EBSD revealed that one void is usually surrounded by several twin boundaries which could act as pinning obstacle (Supplementary figure 4).

\section{Conclusion}

The solid state dewetting behavior of a self-passivated mazed bicrystalline Al thin film model system was investigated in-situ in an ESEM. In-situ annealing experiments in reducing atmosphere at $\sim 823 \mathrm{~K}$ led to rapid solid state dewetting and the formation of $\mu \mathrm{m}$-sized features. In contrast, in oxidizing atmosphere the surface oxide is stabilized, surface diffusion suppressed and dewetting slowed down, which takes place by formation and growth of voids in the Al film underneath the oxide capping layer via interface diffusion. Faceted void formation and growth were observed at $\sim 773 \mathrm{~K}$ in $30 \mathrm{~Pa} \mathrm{O}_{2}$ atmosphere and the bicrystalline $\{111\} \pm<\overline{1} 10>_{A l} \|(0001)<10 \overline{1} 0>_{A l_{2} O_{3}} 50 \mathrm{~nm}$ thick Al film kept its orientation.

Void growth dynamics is influenced by the pinning events of the void|film|substrate triple line. It can be described by a power law $r_{\text {void }} \sim t^{x}$ with an exponent $x$ varying in the range of 0.10 to 0.55 . The void growth dynamics is discontinuous due to pinning which leads to two mechanisms: discontinuous film retraction normal to facets and microkink-flow. The film retraction can be described by an apparent diffusion constant in the range of $\leq 2 \cdot 10^{-15} \frac{\mathrm{m}^{2}}{\mathrm{~s}}$. Fingers may form at pinning sites. With time, depinning occurs and dewetting continues or the fingers pinch-off and form elongated islands as observed in some of the dewetted areas. Such events affect the growth of the void shapes since depinning requires sufficient 
incubation time. As a consequence of local pinning, the void shapes deviate from an ideal hexagon.

Microkink-flow was observed as a new mechanism and occurs along the facets of the void causing a stepwise film retraction normal to the facet. Microkinks are related to faceting instabilities which are intermediate steps in cases where straight facets again evolve. They also result from the combination of multiple microkinks and pinning.

In summary, the in-situ annealing ESEM experiments confirmed the proposed discontinuous void formation mechanism for surface-passivated Al thin films on sapphire [8] and resolved unexpected mechanisms and kinetics. This study shows the potential of in-situ annealing experiments in the ESEM under well-defined atmospheres and controlled temperature conditions for resolving time-dependent details of solid state dewetting.

\section{Acknowledgement}

The authors thank Ilsemarie Lakemeyer for experimental support with the thin film growth. Helpful discussions with Wayne Kaplan are highly appreciated.

\section{References}

[1] P. Jacquet, R. Podor, J. Ravaux, J. Teisseire, I. Gozhyk, J. Jupille, R. Lazzari, Grain growth: The key to understand solid-state dewetting of silver thin films, Scr. Mater. 115 (2016) 128-132.

[2] F. Niekiel, S.M. Kraschewski, P. Schweizer, B. Butz, E. Spiecker, Texture evolution and microstructural changes during solid-state dewetting: A correlative study by complementary in situ TEM techniques, Acta Mater. 115 (2016) 230-241.

[3] G. Dehm, J.M. Howe, J. Zweck, In-situ Electron Microscopy: Applications in Physics, Chemistry and Materials Science, Wiley-VCH Verlag \& Co, Weinheim, Germany, 2012.

[4] S.H. Oh, M.F. Chisholm, Y. Kauffmann, W.D. Kaplan, W. Luo, M. Rühle, C. Scheu, Oscillatory Mass Transport in Vapor-Liquid-Solid Growth of Sapphire Nanowires, Science 330 (2010) 489-493.

[5] Z.-J. Wang, G. Weinberg, Q. Zhang, T. Lunkenbein, A. Klein-Hoffmann, M. Kurnatowska, M. Plodinec, Q. Li, L. Chi, R. Schloegl, M.-G. Willinger, Direct Observation of Graphene Growth and Associated Copper Substrate Dynamics by in Situ Scanning Electron Microscopy, ACS Nano 9 (2015) 1506-1519.

[6] C.V. Thompson, Solid-State Dewetting of Thin Films, Annu. Rev. Mater. Res. 42 (2012) 399-434.

[7] P. Cao, P. Bai, A.A. Omrani, Y. Xiao, K.L. Meaker, H.-Z. Tsai, A. Yan, H.S. Jung, R. Khajeh, G.F. Rodgers, Y. Kim, A.S. Aikawa, M.A. Kolaczkowski, Y. Liu, A. Zettl, K. Xu, M.F. Crommie, T. Xu, Preventing Thin Film Dewetting via Graphene Capping, Adv. Mater. (Weinheim, Ger.) 29 (2017) 1701536.

[8] S.W. Hieke, G. Dehm, C. Scheu, Annealing induced void formation in epitaxial Al thin films on sapphire $\left(\alpha-\mathrm{Al}_{2} \mathrm{O}_{3}\right)$, Acta Mater. 140 (2017) 355-365.

[9] A. Kosinova, D. Wang, P. Schaaf, A. Sharma, L. Klinger, E. Rabkin, Whiskers growth in thin passivated Au films, Acta Mater. 149 (2018) 154-163.

[10] F.G. Yost, Voiding due to thermal stress in narrow conductor lines, Scripta Metallurgica 23 (1989) 1323-1328.

[11] F.G. Yost, D.E. Amos, A.D. Romig, Stress-driven diffusive voiding of aluminum conductor lines, 27th Annual Proceedings., International Reliability Physics Symposium, 1989. p.193-201. 
[12] N. Kristensen, F. Ericson, J.-Å. Schweitz, U. Smith, Hole formation in thin aluminium films under controlled variation of strain and temperature, Thin Solid Films 197 (1991) 67-83.

[13] S.W. Hieke, B. Breitbach, G. Dehm, C. Scheu, Microstructural evolution and solid state dewetting of epitaxial Al thin films on sapphire $\left(\alpha-\mathrm{Al}_{2} \mathrm{O}_{3}\right)$, Acta Mater. 133 (2017) 356366.

[14] M.A. Korhonen, C.A. Paszkiet, C.Y. Li, Mechanisms of thermal stress relaxation and stress-induced voiding in narrow aluminum-based metallizations, J. Appl. Phys. 69 (1991) 8083-8091.

[15] S. Dutta, H.M. Chan, R.P. Vinci, Sub-Surface Oxidation at the Aluminum-Sapphire Interface During Low-Temperature Annealing, J. Am. Ceram. Soc. 90 (2007) 2571-2575.

[16] S. Dutta, J.M. Biser, R.P. Vinci, H.M. Chan, Solid State Annealing Behavior of Aluminum Thin Films on Sapphire, J. Am. Ceram. Soc. 95 (2012) 823-830.

[17] Y. Sugano, S. Minegishi, H. Sumi, M. Itabashi, In-situ observation and formation mechanism of aluminum voiding, 26th Annual Proceedings Reliability Physics Symposium 1988, 1988. p.34-38.

[18] R. Saxena, M.J. Frederick, G. Ramanath, W.N. Gill, J.L. Plawsky, Kinetics of voiding and agglomeration of copper nanolayers on silica, Physical Review B 72 (2005) 115425.

[19] I. Beszeda, I.A. Szabó, E.G. Gontier-Moya, Morphological evolution of thin gold films studied by Auger electron spectroscopy in beading conditions, Applied Physics A 78 (2004) 1079-1084.

[20] F. Cheynis, E. Bussmann, F. Leroy, T. Passanante, P. Müller, Dewetting dynamics of silicon-on-insulator thin films, Phys. Rev. B 84 (2011) 245439.

[21] E. Jiran, C.V. Thompson, Capillary instabilities in thin films, J. Electron. Mater. 19 (1990) 1153-1160.

[22] E. Jiran, C.V. Thompson, Capillary instabilities in thin, continuous films, Thin Solid Films 208 (1992) 23-28.

[23] B.T. Schaefer, J. Cheung, J.F. Ihlefeld, J.L. Jones, V. Nagarajan, Stability and dewetting kinetics of thin gold films on $\mathrm{Ti}, \mathrm{TiO}_{\mathrm{x}}$ and $\mathrm{ZnO}$ adhesion layers, Acta Mater. 61 (2013) 7841-7848.

[24] S. Jahangir, X. Cheng, H.H. Huang, J. Ihlefeld, V. Nagarajan, In-situ investigation of thermal instabilities and solid state dewetting in polycrystalline platinum thin films via confocal laser microscopy, J. Appl. Phys. 116 (2014) 163511.

[25] J. Mizsei, V. Lantto, In situ AFM, XRD and Resistivity Studies of the Agglomeration of Sputtered Silver Nanolayers, J Nanopart Res 3 (2001) 271-278.

[26] C.M. Müller, R. Spolenak, Microstructure evolution during dewetting in thin Au films, Acta Mater. 58 (2010) 6035-6045.

[27] D. Amram, E. Rabkin, Phase Transformations in Au-Fe Particles and Thin Films: Size Effects at the Micro- and Nano-scales, JOM 68 (2016) 1335-1342.

[28] E.J. Luber, B.C. Olsen, C. Ophus, D. Mitlin, Solid-state dewetting mechanisms of ultrathin $\mathrm{Ni}$ films revealed by combining in situ time resolved differential reflectometry monitoring and atomic force microscopy, Physical Review B 82 (2010) 085407.

[29] P. Jacquet, R. Podor, J. Ravaux, J. Lautru, J. Teisseire, I. Gozhyk, J. Jupille, R. Lazzari, On the solid-state dewetting of polycrystalline thin films: Capillary versus grain growth approach, Acta Mater. 143 (2018) 281-290.

[30] S.A. Jang, H.J. Lee, C.V. Thompson, C.A. Ross, Y.J. Oh, Crystallographic analysis of the solid-state dewetting of polycrystalline gold film using automated indexing in a transmission electron microscope, APL Mater. 3 (2015) 126103.

[31] F. Niekiel, P. Schweizer, S.M. Kraschewski, B. Butz, E. Spiecker, The process of solid-state dewetting of $\mathrm{Au}$ thin films studied by in situ scanning transmission electron microscopy, Acta Mater. 90 (2015) 118-132.

[32] Z.-J. Wang, J. Dong, Y. Cui, G. Eres, O. Timpe, Q. Fu, F. Ding, R. Schloegl, M.-G. Willinger, Stacking sequence and interlayer coupling in few-layer graphene revealed by in situ imaging, Nature Communications 7 (2016) 13256.

[33] C.A. Schneider, W.S. Rasband, K.W. Eliceiri, NIH Image to ImageJ: 25 years of image analysis, Nat. Methods 9 (2012) 671-675. 
[34] E. Meijering, FeatureJ: An ImageJ Plugin Suite for Image Feature Extraction, http://imagescience.org/meijering/software/featurej/ (2015).

[35] Q. Tseng, I. Wang, E. Duchemin-Pelletier, A. Azioune, N. Carpi, J. Gao, O. Filhol, M. Piel, M. Thery, M. Balland, A new micropatterning method of soft substrates reveals that different tumorigenic signals can promote or reduce cell contraction levels, Lab Chip 11 (2011) 2231-2240.

[36] L.A. Giannuzzi, F.A. Stevie, A review of focused ion beam milling techniques for TEM specimen preparation, Micron 30 (1999) 197-204.

[37] M. Schaffer, B. Schaffer, Q. Ramasse, Sample preparation for atomic-resolution STEM at low voltages by FIB, Ultramicroscopy 114 (2012) 62-71.

[38] R.M. Langford, M. Rogers, In situ lift-out: Steps to improve yield and a comparison with other FIB TEM sample preparation techniques, Micron 39 (2008) 1325-1330.

[39] L. Jones, H. Yang, T.J. Pennycook, M.S.J. Marshall, S. Van Aert, N.D. Browning, M.R. Castell, P.D. Nellist, Smart Align-a new tool for robust non-rigid registration of scanning microscope data, Advanced Structural and Chemical Imaging 1 (2015) 8.

[40] G. Dehm, B.J. Inkson, T. Wagner, Growth and microstructural stability of epitaxial Al films on (0001) $\alpha-\mathrm{Al}_{2} \mathrm{O}_{3}$ substrates, Acta Mater. 50 (2002) 5021-5032.

[41] S. Schmidt, W. Sigle, W. Gust, M. Rühle, Gallium segregation at grain boundaries in aluminium, Z. Metallkd. 93 (2002) 428-431.

[42] W. Hu, D.A. Molodov, B. Schönfelder, L.S. Shvindlerman, G. Gottstein, HRTEM Study on $\Sigma 7$ Grain Boundary in Aluminium Bicrystals with and without Ga Doping, Interface Science 8 (2000) 335-349.

[43] D. Amram, L. Klinger, N. Gazit, H. Gluska, E. Rabkin, Grain boundary grooving in thin films revisited: The role of interface diffusion, Acta Mater. 69 (2014) 386-396.

[44] D. Amram, E. Rabkin, On the role of $\mathrm{Fe}$ in the growth of single crystalline heteroepitaxial Au thin films on sapphire, Acta Mater. 61 (2013) 4113-4126.

[45] S. Ramanathan, B.M. Clemens, P.C. McIntyre, U. Dahmen, Microstructural study of epitaxial platinum and Permalloy/platinum films grown on (0001) sapphire, Philosophical Magazine A 81 (2001) 2073-2094.

[46] T. Radetic, C. Ophus, D.L. Olmsted, M. Asta, U. Dahmen, Mechanism and dynamics of shrinking island grains in mazed bicrystal thin films of Au, Acta Mater. 60 (2012) 7051 7063.

[47] V. Laurent, D. Chatain, C. Chatillon, N. Eustathopoulos, Wettability of monocrystalline alumina by aluminium between its melting point and $1273 \mathrm{~K}$, Acta Metallurgica 36 (1988) 1797-1803.

[48] G. Levi, W.D. Kaplan, Oxygen induced interfacial phenomena during wetting of alumina by liquid aluminium, Acta Mater. 50 (2002) 75-88.

[49] E. Shaffir, Y. Kauffmann, I. Riess, Void formation in gold films on yttrium-doped zirconia in the initial stage of de-wetting, Acta Mater. 79 (2014) 59-65.

[50] E. Shaffir, I. Riess, W.D. Kaplan, The mechanism of initial de-wetting and detachment of thin Au films on YSZ, Acta Mater. 57 (2009) 248-256.

[51] C.M. Müller, R. Spolenak, Dewetting of Au and AuPt alloy films: A dewetting zone model, J. Appl. Phys. 113 (2013) 1-13.

[52] T.S. Lundy, J.F. Murdock, Diffusion of $\mathrm{Al}^{26}$ and $\mathrm{Mn}^{54}$ in Aluminum, J. Appl. Phys. 33 (1962) 1671-1673.

[53] H. Mehrer, N. Stolica, N.A. Stolwijk, 2.2.13 Aluminum group metals: Datasheet from Landolt-Börnstein - Group III Condensed Matter - Volume 26: "Diffusion in Solid Metals and Alloys" in SpringerMaterials (http://dx.doi.org/10.1007/10390457_22), In: Mehrer H, (Ed.), Landolt-Börnstein - Group III Condensed Matter: Springer-Verlag Berlin Heidelberg.

[54] A. Brandes, G.B. Brooks, Smithells Metals Reference Book, 7th ed, ButterworthHeinemann, Oxford, 1992.

[55] D. Amram, L. Klinger, E. Rabkin, Anisotropic hole growth during solid-state dewetting of single-crystal Au-Fe thin films, Acta Mater. 60 (2012) 3047-3056.

[56] J. Ye, C.V. Thompson, Anisotropic edge retraction and hole growth during solid-state dewetting of single crystal nickel thin films, Acta Mater. 59 (2011) 582-589. 
[57] E. Bussmann, F. Cheynis, F. Leroy, P. Müller, O. Pierre-Louis, Dynamics of solid thinfilm dewetting in the silicon-on-insulator system, New Journal of Physics 13 (2011) 043017. [58] R.V. Zucker, G.H. Kim, W. Craig Carter, C.V. Thompson, A model for solid-state dewetting of a fully-faceted thin film, C. R. Phys. 14 (2013) 564-577. 


\section{Appendices}

\section{A1 Supplementary figures}

Supplementary figure 1: a,b) The full region of interest observed in-situ containing three voids after selected annealing times $\left(\sim 40 \mathrm{~min}, \mathrm{O}_{2}, \sim 773 \mathrm{~K}\right.$. The sequence for void 1 is shown in

Figure 1 and the full dataset can be seen in the Supplementary movie 1). c) Increase of the equivalent void radius with annealing time for the three analyzed voids 1-3 of the dataset and the corresponding power law fits.

Supplementary figure 2: Plan-view SEM micrograph after annealing revealing faceting instabilities as well as a finger ( $\left.\sim 40 \mathrm{~min}, \mathrm{O}_{2}, \sim 773 \mathrm{~K}\right)$.

Supplementary figure 3: Plan-view SEM micrograph revealing the microstructure after $3 \mathrm{~h}$ insitu annealing in $\mathrm{H}_{2} / \mathrm{N}_{2}$ atmosphere at $823 \mathrm{~K}$.

Supplementary figure 4: EBSD orientation maps revealing the in-plane orientation of the $\mathrm{Al}$ thin film containing only $\mathrm{OR}$ a/b and the presence of faceted voids (left: $\sim 40 \mathrm{~min}, \mathrm{O}_{2}, \sim 773 \mathrm{~K}$, right: $\sim 15.3 \mathrm{~h}, \mathrm{O}_{2}, \sim 773 \mathrm{~K}$; OR a/b in purple, voids in bright blue, $\Sigma 3$ twin boundaries in white, area not containing indexed $\mathrm{Al}$ or $\alpha-\mathrm{Al}_{2} \mathrm{O}_{3}$ grains in black).

Supplementary figure 5: Plan-view SEM micrograph after annealing, before binarisation and subsequent void size analysis $\left(\sim 15.3 \mathrm{~h}, \mathrm{O}_{2}, \sim 773 \mathrm{~K}\right)$. Note: the original image size was binned for publication.

Supplementary figure 6: Plan-view SEM micrograph of the selected region of interest for sitespecific TEM analysis (marked by a dashed line) shown in Figure 9.

\section{A2 Supplementary movies}

Supplementary movie 1 The short-term dataset presented as movie ( 40 min in-situ annealing, $\mathrm{O}_{2}$, $773 \mathrm{~K}, 5 \mathrm{fps}, 17.7 \mathrm{~s}$ per SEM frame, scale bar $\left.3 \mu \mathrm{m}\right)$. Note: Charging effects due to the insulating sapphire substrate are visible in the raw data. The presented raw data was only corrected for drift.

Supplementary movie 2 The long-term dataset shown as movie ( $15.3 \mathrm{~h}$ in-situ annealing, $\mathrm{O}_{2}, \sim 773 \mathrm{~K}, 5 \mathrm{fps}, 35.4 \mathrm{~s}$ respectively $88.5 \mathrm{~s}$ per SEM frame, scale bar $\left.5 \mu \mathrm{m}\right)$. Note the change of the time per SEM frame after 205 frames. Note: Charging effects due to the insulating sapphire substrate are visible in the raw data. The presented raw data was only corrected for drift. 